\title{
Vorbemerkung: Die historische Aussage in der Geschichtsschreibung
}

Eine Gesellschaft, die bereit ist, ihre Zukunft aktiv zu gestalten, ist wesentlich auf die Geschichtsschreibung angewiesen. Nachhaltige Veränderungen kommen nämlich nicht zustande durch eine verkrampfte Jagd nach Modernisierung, sondern durch eine stetige und unverkrampfte Auseinandersetzung mit der Vergangenheit. Auf diesem Wege ändert sich das Selbstverständnis, und neue Perspektiven für Denken und Handeln eröffnen sich. Das gilt für den Einzelnen, wie für eine Wissenschaft, wie für eine ganze Gesellschaft. Das vorliegende Buch beschäftigt sich mit der Geschichte der deutschen Sprache. Aber es ist kein Plädoyer für mehr sprachgeschichtliches Wissen in einer vermeintlichen Wissensgesellschaft, sondern eines für eine nicht abreißende Geschichts- und Sprachgeschichtsschreibung. Auf der zweiten Metaebene der Geschichte (Reichmann 1998, 2) werden 25 Sprachgeschichten des Deutschen behandelt, die ihrerseits die deutsche Sprachgeschichte als angeblich gewesenes Geschehen zum Gegenstand haben. Es geht darum zu erläutern, wie die Sprach-/Historiographie in diesen Texten als Medium historischer Erkenntnis, Selbsterkenntnis und gesellschaftlicher Einflussnahme funktioniert.

Damit ist zugleich gesagt: Die Aufgabe der Sprach-/Geschichtsschreibung besteht nicht im Wesentlichen darin, über vergangene Geschehnisse zu informieren. Historiker wuchern mit dem Pfund der Information und der Fülle der zugänglichen Überlieferungsdaten. Historiographen hingegen arbeiten - auf dem Wege der Information über die Vergangenheit - an ihren eigenen historischen Begriffen und an den mit diesen Begriffen verbundenen, für sie wichtigen historischen Erfahrungen: an ihrem Verständnis von Krieg und Frieden, von Toleranz und Intoleranz, von guter Sprache und schlechtem Stil, vom Eigenen und vom Andersartigen, von Dialekt und Hochsprache, vom Deutschen in Deutschland und vom Deutschen in Österreich usw. usf. Kluges sprachliches Handeln ist hier gefragt (Hermanns 1987, 76f.), denn die Geschichtsschreibung ist eine einzige Gratwanderung: Schreiben über die Vergangenheit und zugleich Schreiben dafür, dass wir uns mit unseren Begriffen in der Gegenwart und Zukunft besser zurechtfinden. Insofern ist die historische Aussage keine theoretische oder narrative Aussage, sondern eine kritische Aussage. Die Autoren dieser Aussagen sind keine Theoretiker (wie die begrifflich subsumierenden Historiker und Sprachhistoriker), und sie sind auch keine Künstler (wie ein erzählender Romancier). Sie sind sprachliche Handwerker, die weniger am Ausdruck einer Botschaft arbeiten als an ihrem Medium. 
Mit der historischen Aussage als zentralem Gegenstand schließt die Untersuchung an die Geschichtswissenschaften an (Veyne 1971/1996; 1990; Koselleck 1997/ 2010; Faber 51982; Rothermund 1994), auch an die (analytische) Geschichtsphilosophie (Danto 1965; 1974; Baumgartner ${ }^{3} 1975$; Ricœur ${ }^{2} 2007$ ), die Literaturwissenschaft (Schiffer 1980; Jauß 1982) und die Sprachwissenschaft (Stempel 1973a). Im Hintergrund steht das im Ursprung textlinguistische Anliegen zu klären, inwiefern die historische Aussage die Geschichte/Sprachgeschichte als kohärente Texteinheit und Textsorte im Deutschen zusammenhält. Medientheoretisch gewendet betrifft dieses Anliegen aber nicht die Frage nach der Transphrastik, dem Textthema, der Textfunktion bzw. Autorabsicht (Schlieben-Lange 1983, 27 f.; Reichmann 1996; Heinemann 2000b; Brinker ${ }^{7} 2010$; Adamzik ${ }^{2} 2016$ ), sondern die Frage nach dem Medium, welches die Geschichtsschreibung trägt (Groys 2000). Die zentrale These lautet: Das Medium der Geschichtsschreibung ist die historische Aussage, und deren wichtigstes Spezifikum besteht darin, mit der syntaktisch, semantisch und pragmatisch flexiblen, multifunktionalen Präpositionalgruppe mit für gebildet zu werden. Denn Historiographen sprechen nicht von der Bedeutung vergangener Ereignisse schlechthin, sondern sie sprechen davon, welche Relevanz und Bedeutung die Ereignisse der Vergangenheit für etwas anderes haben (Veyne 1990, 51). In der Präpositionalgruppe erscheinen dabei wertbesetzte begriffliche Größen, die semantisch und pragmatisch der Gegenwart des Historiographen angehören. Das aber heißt: Indem die Historiographen ihre historischen Aussagen systematisch mit der Präpositionalgruppe mit für bilden, sprechen sie über die Vergangenheit und verhandeln zugleich ihre Gegenwart.

Als eine der wichtigsten kommunikations- und sprachtheoretischen Vorannahmen der Untersuchung gilt, dass überall dort, wo miteinander und (besonders in Schrifttexten) füreinander gesprochen wird, auch die Bedingungen dieses Mit- und Füreinander-Sprechens mit reflektiert und ausgehandelt werden. Diese (Selbst-) „Reflexivität“ (Schlieben-Lange 1975, 192f.) bzw. „rekursive Transkriptivität“ (Jäger 2005, 48) kann man in der Historiographie immer dort beobachten, wo die Präpositionalgruppe mit für im Textverlauf syntaktisch verrückt, semantisch verschoben und pragmatisch immer neuen kommunikativen Kontexten anverwandelt wird. Dadurch wird Geschichte für je andere Lesergruppen und ihre je andere begriffliche Gegenwart in einem jeweils anderen selbstreflexiven Verständnis rekursiv je anders geschrieben - derart, dass sich Strukturen und Funktionen der historischen Aussage im Textverlauf ständig ändern und damit auch die Ereignisbedeutungen. Darin besteht das Handwerk der Historiographen: Durch ihren handwerklichen Umgang mit der Präpositionalgruppe im Aussage-, Text- und Kommunikationszusammenhang schaffen die Historiographen Sprachgeschichte, und sie reflektieren, wie sie das tun. „Die verrückte historische Aussage mit für" bildet deshalb das Thema und Leitmotiv der Untersu- 
chung, mit der Textlinguisten, Sprachhistoriker, Historiker und Geschichtstheoretiker gleichermaßen angesprochen werden sollen (Blumenthal 2000; Trabant 2005; Langer/Davies/ Vandenbussche 2012; Kansteiner 2018).

Für eine Übersicht über die Ergebnisse der Hauptkapitel 1 bis 5 sei auf die Zusammenfassung in Kapitel 6 verwiesen. Eine Methodendiskussion ist in Kapitel $1.7 \mathrm{zu}$ finden; zahlreiche Belege, insbesondere für die Analysen in Kapitel 4 und 5 auf der Internetseite des Verlags (https://www.degruyter.com/view/ title/578691). An dieser Stelle sei es mir erlaubt, ein einführendes Beispiel zu nennen, anhand einer historiographischen Arbeit, die mit der deutschen Sprachgeschichte gar nichts zu tun hat, die zudem auch noch in Französisch verfasst ist, die mir aber in den vergangenen Jahren eine leidige Erfahrung erleichtert hat: Die Begriffe vom Kind, von der Familie und vom Familienleben, die im Alltag für viele zentral und handlungsleitend sind, sind in demselben Alltag kaum reflexionsfähig. Praktische Zwänge und konventionalisierte Berührungsängste bewirken, dass im halböffentlichen Gespräch auf diese Begriffe nur reagiert wird und ein kritischer und souveräner Umgang mit ihnen kaum möglich ist. Die „Geschichte der Kindheit“ von Philippe Ariès (1975; 1988), in der an genau diesen Begriffen gearbeitet wird, ist ein passender Anlass, solche Reflexionen dennoch auch im Alltag in Gang zu bringen. Man spricht über die Vergangenheit und verhandelt trotzdem seine Gegenwart.

Dasselbe tut der Historiograph: In einem sehr schwierigen Absatz im Text zitiert Ariès ausführlich aus dem (von Héroard verfassten) leibärztlichen Tagebuch über die Kindheit Ludwigs XIII., und er bemerkt: „Der moderne Leser“ dieses Tagebuchs sei verwirrt von der Freiheit/Freizügigkeit, mit der man die Kinder damals behandelte: Il „est confondu de la liberté avec laquelle on traitait les enfants“ (Ariès 1975, 141). Es handelt sich um einen schwer darstellbaren Ausschnitt aus der Vergangenheit, denn vom ersten bis zum siebten Lebensjahr wurde der Junge „zum Spaß“ fortlaufend im Intimbereich belästigt. (Danach setzte die Erziehung ein, mit 14 Jahren wurde er verheiratet). Indem Ariès sich (mit genau diesem Satz) an „den modernen Leser“ wendet, antizipiert er, in welcher Weise dieser Sachverhalt diesen Leser betrifft und wie der ihn beurteilt, wie der Sachverhalt für den Leser wirkt und was er für ihn bedeutet. Ariès unterlässt es dabei, die Präposition für (pour) auszubuchstabieren. Und doch ist sie in den Hauptprädikaten dieses Satzes impliziert: Es geht bei dem, was er im Kontext „vulgäre Scherze“ und „anstößige/unschickliche“ Handlungen nennt (ebd.), um eine „Freiheit“ bzw. „Freizügigkeit“, die für den Leser „verwirr“end ist. Mit dieser Interpretation bezieht sich der Historiograph auf die überlieferte Vergangenheit, er bezieht sich aber in gleichem Maße auf diesen „modernen Leser“. Auch über ihn und über seine Erfahrungen im Umgang mit Kindern spricht der Historiograph. 
Das Verständnis dieser Aussage wie des ganzen Absatzes hängt nun ganz und gar davon ab, für wen und für was genau der Historiograph diese „verwirrende Freiheit/Freizügigkeit“ geltend macht, worüber er also, während er über das Tagebuch und den kleinen König spricht, zudem noch spricht. „Der moderne Leser“ ist ja nur ein Platzhalter. Sind denn diese „Scherze“ in den Augen des Historiographen eine „Freiheit“, die als naive, ursprüngliche, „natürliche“ Ungezwungenheit den Erwachsenen und Kindern damals zukam (dem modernen Leser aber nicht)? Oder sind sie eine „schockierende, unerträgliche Freizügigkeit“ und eine Frechheit (,audace“) für die Vertreter der (erst im 18. Jh. und 19. Jh. kultivierten) „strengen bürgerlichen Moral“? Handelt es sich vielleicht um eine positive Handlungsfreiheit für all diejenigen, die dieses Verhalten so „natürlich“ finden, dass sie sich über die bürgerliche Moral lieber hinwegsetzen und diese Freiheit für sich reklamieren? Oder handelt es sich doch um einen ,unerträglichen“ Übergriff, um eine „Freiheit“, die sich die Erwachsenen damals zum Schaden der Kinder herausnahmen? Für all diese historischen Aussagen mit für gibt es bei Ariès Kontextindikatoren; und indem der Historiograph sich an „den modernen Leser“ wendet, entfaltet er eine für einen Geschichtstext beachtliche Vielfalt von Redegegenständen, die lauter Personen- und Interessengruppen aus seiner eigenen Gegenwart umfasst. Bewerkstelligt wird das mit dem überaus ambivalenten Prädikat der liberté, das im Kontext dieses Absatzes nach Maßgabe verschiedener - wenn auch nicht ausgesprochener - Präpositionalgruppen mit für ständig neu und mit neuen Prädikaten interpretiert wird. Auf diese Weise wird die Präposition für wieder und wieder verrückt, in diesem präzisen Fall semantisch verschoben (für die modernen Leser - für die erwachsenen Leser - für die Erwachsenen damals - für die naiven Erwachsenen damals - für die naiven Erwachsenen heute ...). Solche Manipulationen sind in der Historiographie unerlässlich, denn sie garantieren beides: dass die Vergangenheit nicht abschließend beurteilt und in einer einzigen Schublade der Erinnerung versenkt wird und dass die kritische Arbeit an den eigenen Erfahrungsbegriffen aufrechterhalten wird. Indem der Historiograph die Präpositionalgruppe mit für dauernd neuplatziert, kann er, im Rahmen dieser Präpositionalgruppe, nach und nach bestimmte Erfahrungen und Interessen der Gegenwart in den Blick nehmen, und er kann die Vergangenheit einmal so und einmal anders beurteilen.

Freilich entspricht das Verfahren nicht dem Ideal neutralen, wissenschaftssprachlichen Formulierens, denn die Rede von einer Freiheit/Freizügigkeit impliziert eine Bewertung. Die kommt vor allem dadurch zustande, dass die Freiheit/ Freizügigkeit, die der Historiograph für eine Person/-engruppe geltend macht, für diese immer auch einen Vorteil bedeutet, gegebenenfalls auch einen Nachteil für eine andere. Indem er also genau dieses und kein anderes Prädikat verwendet und indem er es affirmativ (als Freiheit und Ungezwungenheit) oder tadelnd (als 
Freizügigkeit, Frechheit oder Übergriff) verwendet, begünstigt er eine Größe und benachteiligt er eine andere. Historiographen treffen deshalb nicht nur Aussagen über Vergangenheit und Gegenwart, sondern sie ergreifen zugleich Partei. Mit der (elliptischen) Rede von der liberté allein vollzieht Philippe Ariès verschiedene Sprechakte: Für die naiven Erwachsenen (der Vergangenheit und der Gegenwart) bittet er um Verständnis, für die (Spieß-) Bürger hält er eine Provokation bereit; denjenigen, die gegen die bürgerliche Norm aufbegehren, gibt er ein legitimierendes Argument an die Hand; und für all diejenigen, die für die Kinder Respekt haben und Respekt einfordern, räumt er ein: An diesem historisch belegten Beispiel sieht man, dass es auch andere Positionen gibt. Er redet über all diese Gruppen von Betroffenen, über ihre Eindrücke, Urteile, Erfahrungen und Interessen, aber er tut das nicht irgendwie, sondern derart, dass er ihnen eine Freiheit/ Freizügigkeit an die Hand gibt, jeder von ihnen $\mathrm{zu}$ einem mehr oder weniger großen Gewinn, zu einer mehr oder weniger willkommenen Verfügung, zu einem mehr oder weniger gewichtigen Schaden. Die Präposition für wird dabei zu einem der wichtigsten Bedeutungsträger im Text der Geschichte, denn weil die Historiographen mit dieser Präposition sprachlich arbeiten müssen, müssen sie parteilich sein. Allein das Verfahren der im Textverlauf immer neu verrückten historischen Aussage stellt sicher, dass sie nicht - wie Ideologen, Anwälte, Lobredner - einseitig parteilich sein müssen. So begründet nicht Unparteilichkeit, sondern differenzierte Parteilichkeit ihren Anspruch auf wissenschaftliche Rationalität. Indem Ariès die Präposition für immer wieder neuplatziert, muss er keine unverrückbaren Wahrheiten und Normen verkünden, aber er kann, während er sich adäquat über seine Quelle äußert, zugleich sehr effektiv zu einer kritischen Reflexion bestehender Handlungsnormen herausfordern.

Verfolgt man nun die illokutionäre (nicht mehr „nur“ semantische) Neuplatzierung der Präpositionalgruppe, dann liegt es auf der Hand, dass der Historiograph die Naiven und die Mutwilligen viel mehr begünstigt als die Strengen und Kompromisslosen. Für die Kinder tut er überhaupt nichts. Im Gegenteil: Er vertraut seiner Quelle blind. Hier wie da wird so viel „gelacht“ (alles geschah „par plaisanterie“), dass man meinen könnte, für ein Kind bestände die größte Freiheit darin, über körperliche Intimitäten zu lachen. Seine Geschichte der Kindheit, vom „Kind und dem Familienleben im Ancien Régime“ (Ariès 1975; 1988) ist deshalb eine Geschichte, in der sich nur die Erwachsenen einigermaßen vielseitig spiegeln können. Der Begriff vom Kind ist demgegenüber ausgesprochen unterkomplex.

Wo es einen solchen blinden Fleck in einem Geschichtsbuch gibt, endet die Geschichtsschreibung, und es beginnt eine Geschichtsideologie. Ariès kann die Interessen der Kinder nicht berücksichtigen, denn er macht sich letztlich doch zum Anwalt einer einzigen Parteisache. Als Historiker hat er eine Mission. Er benutzt seine Quelle, um sich für die historisch-kulturelle Relativität starkzumachen. Er tut 
dies explizit, einseitig und kategorisch. Der moderne Leser ist für ihn einer, der im Gefängnis der Gegenwart (im Frankreich der 60er und Anfang der 70er Jahre) eingeschlossen ist, weil er nichts anderes kennt als den strengen bürgerlichen Umgangskodex mit moral, décence, réserve, discipline, éducation usw. Diesen Leser schockiert er mit der Kindheit des kleinen Königs, und denjenigen Leser, der dafür offen wäre, aus seinem Gefängnis auszubrechen, den verwirrt und überrascht er, um ihm die Gelegenheit zum Ausbruch zu geben. Die Freiheit, die er in dem Tagebuch Héroards findet, widmet er diesen Lesern, damit sie sich befreien.

Er verrennt sich dabei, und zwar nicht nur moralisch: Man könne sich schon vorstellen, „was der moderne Psychoanalytiker dazu sagen würde! Doch hätte dieser Psychoanalytiker unrecht. Die Einstellung zur Sexualität und zweifellos auch die Sexualität selbst“ sei „von Milieu zu Milieu und infolgedessen auch von Epoche zu Epoche und von Mentalität zu Mentalität verschieden“ (ebd. 179). Dort, wo der Historiograph diesen Zeigefinger erhebt, scheitert er historiographisch. Der handwerklich gearbeitete und kluge Umgang mit der Präposition für hört an dieser Stelle auf, weil er hier einseitig nur für eine einzige Parteisache eintritt und dabei mindestens einen anderen Gesichtspunkt explizit ins Unrecht setzt und diffamiert. Die historisch-kulturelle Relativität mag ihm wichtig sein, aber auch als guter Historiker mit einem ernsthaften Anliegen muss er sie nicht gegen einen anderen Standpunkt ausspielen. Dass es dabei ausgerechnet um den Standpunkt der Psychoanalytiker geht, die solche „Scherze“ für absolut unangebracht halten, ist mindestens so schwierig wie das Tagebuch von Héroard und müsste selbst (in einer Geschichte der Geschichtsschreibung) zum Gegenstand verrückter historischer Aussagen gemacht werden.

Auch in der Sprachgeschichtsschreibung wird die Präposition für gegenwärtig häufig so platziert, dass die Historiographen die Überlieferungsdaten für die Zwecke der Sprachgeschichte allein benutzen und geltend machen. Aussagen über die ganze deutschsprachige Überlieferung sind zugleich Aussagen nur für die professionellen Sprachhistoriker und ihre Anliegen. Vielleicht ist das ein Grund dafür, dass die Begriffe von der Sprache, von der deutschen Sprache und der (deutschen) Sprachgeschichte im halböffentlichen Alltag einen ähnlich unaufgeklärten Status haben wie die von Familie und Kind. Sprachreflexion und Sprachgeschichtsreflexion finden hier so gut wie nicht statt (Antos 2003, 471).

Dieser unbefriedigenden Situation kann man beikommen, indem man die syntaktischen, semantischen und pragmatischen Gebrauchsregeln der Präposition für in der Sprachgeschichtsschreibung untersucht und diese dann auch kritisch interpretiert. Durch das Studium der verrückten historischen Aussage mit für können die Historiographen ein Verhältnis zu ihrem parteilichen Umgang mit ihren Gegenständen finden. Nützlich erscheint mir dabei, nicht mit großer Geste nach dem Sinn von Geschichte und Geschichtsschreibung zu fragen (Trabant 
1976b; Harth 1982b; besonders Rüsen 1990), sondern sich auf das sprachliche Material zu konzentrieren und das Identitätsproblem der historischen Zunft auf ein handwerkliches herunterzubrechen. Die Geschichte bedeutet für die Leser in der Gegenwart nicht mehr, aber auch nicht weniger als das, was der Historiograph beim Umgang mit dem für für sich und seine Leser erarbeitet. Gerade weil es sich bei der Präposition für um ein scheinbar unscheinbares und unspektakuläres Zeichen handelt, eignet es sich in besonderer Weise für einen unaufgeregten Zugang zu einem sehr umstrittenen Diskurs.

Bei der Kritik der Sprachgeschichten des Deutschen verzichte ich auf jedes vermeintlich sachliche Argument aus dem Bereich des sogenannten Geschichtswissens und verwende stattdessen eine konsequent linguistische Methode: die Methode der „Deutschen Satzsemantik“ (Polenz ${ }^{2}$ 1988), die zwischen den Zeilen liest und die das, was ein Autor sagt, mittels Paraphrasierung noch einmal sagt anders, umständlicher, einfacher. Wo die Präpositionalgruppe explizit im Text geäußert wird, wird sie umformuliert, wo sie (in einem Wort wie Freiheit) impliziert ist, wird sie explizit gemacht, und wo sie ausgespart wird, wird sie hinzugefügt - derart, dass sie dann umformuliert werden kann. Im Grunde schreibe ich damit die behandelten Sprachgeschichten einfach weiter, denn auch sie reformulieren, verschieben, erneuern, ergänzen und erläutern ihre Präpositionalgruppen mit für. Nutzt man dieses Vorgehen systematisch, dann entsteht keine einzige Situation, in der man der Präpositionalgruppe nur eine einzige Paraphrase bzw. nur eine Funktion und Bedeutung zuweisen könnte. Die scheinbar einfache, nicht hypotaktisch gegliederte Struktur der historischen Aussage (Die vulgären/anstößigen Scherze mit dem kleinen Ludwig XIII. sind für den modernen Leser eine verwirrende Freiheit/Freizügigkeit) korrespondiert nämlich mit der Multifunktionalität ihrer einzelnen Elemente überhaupt nicht. Es ist besonders die Präpositionalgruppe mit für, die mehrere Aufgaben - syntaktische, semantische, pragmatische - übernimmt und die, noch wenn sie elliptisch ausgespart wird, vieles aussagen und vieles bewirken kann. Deshalb muss jedes für doppelt und dreifach aus- und um- und neu formuliert werden. Das Zeichen eröffnet dem Autor wie dem Leser Schreib- und Interpretationsspielräume, und deshalb kann man nicht, wie die Satzsemantik das ursprünglich vorsieht, „herausfinden“, was die Autoren „eigentlich“ sagen woll(t)en. „Zur Klärung sprachlicher Missverständnisse oder Unklarheiten, zur Aufdeckung sprachlicher Verschleierungen“ (Polenz ${ }^{2} 1988$, 29) wird das Buch nichts beitragen, denn demjenigen, der dieses Ziel verfolgt, muss jeder Gebrauch der Präpositionalgruppe missverständlich, rätselhaft und vielleicht sogar verlogen erscheinen. Weil sie sich systematisch gegen eindeutige Funktions- und Bedeutungszuweisungen sperrt, bildet sie beim Lesen „eine unwillkürliche Abweichung vom Programm, eine Regung, eine Bewegung, einen Fehler, einen Ausrutscher“ (Groys 2000, 69). Wo sie den syntak- 
tischen Anschluss zu Freiheit in ganz verschiedenen Bedeutungen oder zu einem anderen ambivalenten Prädikat bildet, wo sie unvermittelt ihre syntaktische Position oder ihre pragmatische Funktion ändert (etwa indem die Freiheit plötzlich zu einem Argument für die historische Relativität und gegen die Psychoanalytiker gemacht wird), erscheint sie dem Leser als ein „unpassendes Zeichen“ und als Teil einer „verrückten Aussage“.

Ihren theoretischen Platz hat die „verrückte Aussage“ in der Medienontologie von Boris Groys (ebd. 70 f.). Hier steht nicht das kommunikativ handelnde Autorsubjekt im Zentrum, sondern das „medienontologische Subjekt“, das mehr eine Projektion des Lesers ist als eine reale Person. Wer einen Text aufmerksam liest, sieht einen anonymen Handwerker bei der Arbeit und wie der dabei ist, sein sprachliches Medium zu traktieren. Dieser Handwerker hat gegenüber der Sprache keine souveräne Position, die es ihm ermöglichen würde, sich eigentlich und eindeutig auszudrücken. Es ist umgekehrt das Medium, welches ihm Möglichkeiten eröffnet und gleichzeitig Grenzen setzt. Für den, der dieses Handwerkersubjekt arbeiten sehen will, sind die geeignetsten Zeichen die uneindeutigen Zeichen und diejenigen Zeichen, die sich den Vereindeutigungs- und Normalitätserwartungen gerade nicht beugen. Insofern ist eine „verrückte Aussage“ mit der Präposition für nicht nur eine, in der diese Präpositionalgruppe (im Verhältnis $\mathrm{zu}$ einer anderen Aussage) neuplatziert und verrückt wird. Es ist auch eine Aussage, die falsch klingt, fou und foolish. Das ist ihr heuristischer Wert: Eine verrückte Aussage „scheint uns zwar meistens falsch zu sein“, aber sie wirkt auch „zugleich aufrichtig, authentisch, offenbarend“ (ebd. 71). Nicht, dass ein Autor damit den „bewussten Entschluss“ verkünden würde, endlich die Wahrheit über sein Inneres zu sagen“ (ebd. 66). Aber dort, wo ein Maler im Überschwang der Inspiration an seinem Farbauftrag arbeitet, kann es passieren, dass er dem Betrachter absichtlich oder unabsichtlich den Blick auf die Leinwand freigibt. Und dort, wo der Historiograph (das submediale Handwerkersubjekt der Historiographie) die Präpositionalgruppe mit für neuplatziert, kann es passieren, dass er dem Leser einen Blick auf sein Medium ermöglicht: auf die historische Aussage.

Als empirische Grundlage dieser nicht corpus-, sondern text(sorten)linguistisch ausgerichteten Studie habe ich 25 sogenannte Sprachgeschichten des Deutschen ausgewählt, die aus der Zeit der Weimarer Republik bis 2016 stammen, mit einem Vergleich noch rückwärtig zu Jacob Grimm 1848. Es handelt sich dabei um ein- oder mehrbändige Monographien, auch einige unselbständige Publikationen, die sich selbst Geschichte der deutschen Sprache oder Deutsche Sprachgeschichte nennen oder die erkennbar dasselbe Textthema verfolgen. Die Auswahl sollte die historische Variabilität der Textsorte respektieren, d.h. bezüglich der sogenannten textexternen und textinternen Faktoren ausgewogen und repräsentativ sein: zeitlich / weltanschaulich gestreut und repräsentativ, mit (kur- 
zen) Überblicksdarstellungen und (umfänglichen) ambitionierten Projekten, mit wissenschaftsgeschichtlich relevanten und weniger relevanten Texten, mit völlig vergessenen und aktuell (mutmaßlich) häufig benutzten Texten, wieder bearbeiteten/mehrfach neu aufgelegten Texten, mit individuell oder von einem Autorenkollektiv verantworteten Texten, auch mit mehreren unterschiedlichen Texten ein und desselben Autors, mit Texten von Autoren, die institutionell an der Universität integriert waren/sind oder nicht. Sprachgeschichten der sogenannten Auslandsgermanistik sind leider kaum repräsentiert, auch keine anderssprachlichen Darstellungen. Sprachgeschichten im Internet wurden ebensowenig berücksichtigt (Bär 2013) wie eine geschlechtsspezifische Differenzierung in Texte weiblicher und männlicher Autoren.

Das Schriftbild wird wie in dieser Vorbemerkung gehandhabt: Nachgewiesene, wörtliche Einzelzitate sind wie üblich mit doppelten Anführungszeichen markiert. Zusätzlich verwende ich die Kursive, um die von mir jeweils schon einzelfallübergreifend verallgemeinerte und gleichwohl zitierte Metasprache der Sprachhistoriographen hervorzuheben, insbesondere auch das für. Denn eine Untersuchung auf der zweiten Metaebene der Geschichte muss die originale Metasprache der untersuchten Texte kenntlich machen und darf diese mit ihrer eigenen Metasprache nach Möglichkeit nicht verwechseln. Man nehme dieses aufwändige optische Verfahren im Fließtext als Kompensation für fehlende Tabellen, Graphiken, Bilder. Im Ganzen habe ich mich um ein konsequent versprachlichendes (paraphrasierendes und argumentierendes) Darstellungsverfahren bemüht, das Erwartungen von Bildlichkeit, schneller Nachschlagbarkeit, Querlesen und anderem Hypertextbedarf gerade nicht entspricht. Lieber wollte ich den Kopf, der im Computer abgeschlagen vor mir lag, (wie Saint Denis) unter dem Arm noch ein Stück weitertragen (Michel Serres: Petite Poussette. Paris. Le Pommier. 2012). Im Übrigen halte ich mich an die Unterscheidung von grammatischem Genus und biologischem Sexus. Meine Leser in ihrer Geschlechtlichkeit als Frauen und Männer anzusprechen, das erschiene mir viel zu anzüglich. Deshalb werden der Leser, der Autor und der Historiograph als Rollenbegriffe behandelt und mit dem für Rollen üblichen maskulinen Genus versehen, ohne dass damit die Frauen aus dem Text ausgesperrt würden. Frauen wie Männer sollen diese Rollen jederzeit gerne einnehmen. 
